\title{
Investigating the Resilience of Unstructured Supernode Networks
}

\author{
Michele Amoretti, Member, IEEE, and Gianluigi Ferrari, Senior Member, IEEE
}

\begin{abstract}
In this letter, we present a novel analytical framework to analyze the resilience of Unstructured Supernode Networks (USNs), where a "leaf" node can be promoted, after a fixed time interval, to the role of "supernode," with nonpreferential attachment to a given number of existing supernodes. In particular, relying on an Absorbing Markov Chain (AMC)based model of a supernode behavior, we derive an efficient approximation of the node degree distribution of an USN. This model also allows to estimate a supernode's probability of isolation. The proposed analytical framework is validated by simulation results.
\end{abstract}

Index Terms-Peer-to-peer, network resilience, Absorbing Markov Chain (AMC).

\section{INTRODUCTION}

$\mathbf{P}$ EER-TO-PEER (P2P) networks are inherently robust against churns, i.e., random departures and arrivals of nodes [1]. The isolation time $T$ is defined as the time before all neighbors of a generic peer are simultaneously in the departed state [2]. The expected isolation time $\mathbb{E}[T]$ is a useful metric to evaluate the resilience of a given $\mathrm{P} 2 \mathrm{P}$ overlay scheme and to compare different replacement strategies.

A P2P overlay network is unstructured if the statistical characterization of the links among peers (being them actual or potential connections) are usually unknown to the peers and are not relevant to their message routing strategies. Unstructured Supernode Networks (USNs) are characterized by a group of peers, denoted as "supernodes," which have the responsibility of routing messages. Conversely, other peers ("leaf" nodes) are only resource providers and consumers, and need to connect to the supernode layer in order to publish and discover resources. A leaf node is promoted, after a proper time span, to the role of supernode. At promotion, it connects to other supernodes, selected either randomly (this will be the case of the current work) or according to a specific strategy. While a supernode can be connected to many other supernodes at the same time, a leaf node usually maintains only one connection with a supernode. The node degree distribution of the whole network can be obtained from those of supernodes and leaf nodes [3].

In this letter, we present a novel analytical framework for the evaluation of the resilience of USNs with non-preferential

Manuscript received February 7, 2013. The associate editor coordinating the review of this letter and approving it for publication was P. Serrano.

M. Amoretti is with the Centro Interdipartimentale SITEIA.PARMA, Università degli Studi di Parma, Parma, Italy (e-mail: michele.amoretti@unipr.it).

G. Ferrari is with the Wireless Ad-hoc and Sensor Networks (WASN) Lab, Dipartimento di Ingegneria dell'Informazione, Università degli Studi di Parma, Parma, Italy (e-mail: gianluigi.ferrari@unipr.it).

The work of G. Ferrari is partially supported by the EU project CALIPSO (grant no. 288879).

Digital Object Identifier 10.1109/LCOMM.2013.043013.130305 attachment at the moment of supernode promotion. In particular, we first show how to model a supernode behavior through an Absorbing Markov Chain (AMC). This is an expedient to derive the node degree distribution of an USN. By denoting as $m$ the number of connections (to other supernodes) created at the moment of promotion of a leaf node to the role of supernode, we evaluate the node degree distribution of supernodes and the probability of a supernode's isolation as functions of $m$. Our analytical results are confirmed by realistic simulations.

\section{Analytical Framework}

\section{A. Continuous Time Domain}

In order to describe USNs and analyze their resilience, we build on the modeling framework introduced in [3]. A network is modeled as an undirected graph, where the node degree, which is the number of links starting from a node, is described, with reference to the whole network, in terms of its Probability Mass Function (PMF):

$$
P(k)=P\{\text { node degree }=k\} \quad k=0,1, \ldots
$$

The topology of the network emerges from the peer dynamics, that are necessarily modeled as stochastic processes, due to the lack of centralized control in the network. Two fundamental churn properties are: (i) the inter-arrival time, i.e., the time interval between the beginning of one session and the beginning of the next session (not necessarily by the same peer); and (ii) the session length $L$, i.e., the time interval between the beginning and the end of a session. The inter-arrival time in BitTorrent peergroups (that are relatively small, including tens to hundreds of peers, depending on the popularity of the shared file) is well modeled by a Weibull distribution [4]. In Gnutella, the exponential distribution is a suitable model [5]. It has also been observed that the distribution of the session length is often heavy-tailed, as most users spend minutes per day browsing the network, while a few other peers exhibit server-like behavior and keep their computers online continuously for weeks [6].

Suppose that a leaf node $v$ joins at time $t_{v}$, and $d_{i}$ is the departure time of the $i$-th neighbor of $v, i \in\left\{0,1, . ., k_{v}\right\}$, where $k_{v}$ is the number of neighbors of node $v$ at the given time. The residual lifetime of neighbor $i$ (at time $t_{v}$ ) is $R_{i}=d_{i}-t_{v}$. According to renewal theory [7], if $F(x)$ is the distribution of the session length $L$, then the distribution of the residual lifetime $R$ is

$$
F_{R}(x)=\frac{1}{\mathbb{E}[L]} \int_{0}^{x}[1-F(y)] \mathrm{d} y .
$$

Let $d$ be the fixed session length after which a node is promoted to the supernode state. The session length $L_{s}=L-$ 


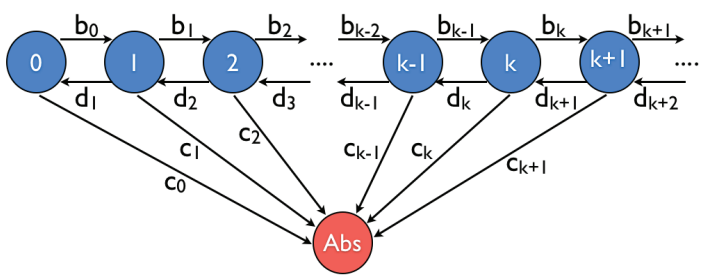

Fig. 1. The AMC that models the evolution of the node degree of a supernode.

$d$ of the a supernode has the following Cumulative Distribution Function (CDF):

$$
\begin{aligned}
F_{s}(x) & =P\left\{L_{s} \leq x\right\}=P\{L-d \leq x \mid L>d\} \\
& =\frac{F(x+d)-F(d)}{1-F(d)} .
\end{aligned}
$$

Theorem 1. Consider $L$ with a shifted Pareto CDF $F(x)=$ $1-(1+x / \beta)^{-\alpha}, x>0, \alpha>1, \beta>0$. Then, the expected value of $L_{s}$ is:

$$
\mathbb{E}\left[L_{s}\right]=\left(1+\frac{d}{\beta}\right) \mathbb{E}[L] .
$$

Proof: Since $L_{s}$ is a non-negative random variable, its expected value can be written as

$$
\mathbb{E}\left[L_{s}\right]=\int_{0}^{+\infty}\left[1-F_{s}(x)\right] \mathrm{d} x .
$$

Using (2), the latter becomes

$$
\begin{aligned}
\mathbb{E}\left[L_{s}\right] & =\left(1+\frac{d}{\beta}\right)^{\alpha} \int_{0}^{+\infty}\left(1+\frac{x+d}{\beta}\right)^{-\alpha} \mathrm{d} x \\
& =\frac{\beta}{\alpha-1}\left(1+\frac{d}{\beta}\right)
\end{aligned}
$$

which, owing to the fact that $\mathbb{E}[L]=\beta /(\alpha-1)$, proves (3).

Let us now focus on a scenario of particular interest. Suppose that all nodes are initially in leaf state. As already introduced above, if the lifetime $L$ of a node is longer than $d$, then at time $d$ the leaf node becomes a supernode and, according to a non-preferential attachment rule, connects to $m$ other randomly selected supernodes. In next subsection, we show that the evolution of the node degree of a supernode can be modeled by means of an embedded AMC, where each state corresponds to a different value of the node degree.

\section{B. Embedded $A M C$}

An illustrative representation of the AMC model of a supernode evolution is shown in Fig. 1. The generic state $k$ (corresponding to $k$ connections to other supernodes) can change upon: a neighbor departure, corresponding the neighbor's death $(k \rightarrow k-1)$; a neighbor arrival $(k \rightarrow k+1)$; or the supernode death, modeled as the entrance into the unique absorbing state $(k \rightarrow \mathrm{A})$.

The transition matrix of any AMC can be written in the following canonical form [8]:

$$
\mathbf{P}=\left(\begin{array}{ll}
\mathbf{I} & \mathbf{R} \\
\mathbf{0} & \mathbf{Q}
\end{array}\right)
$$

where: $\mathbf{I}$ is an $r$-by- $r$ identity matrix, with $r$ being the number of absorbing states; $\mathbf{0}$ is an $r$-by- $q$ zero matrix, with $q$ being the number of transient states; $\mathbf{R}$ is a nonzero $q$-by- $r$ matrix; and $\mathbf{Q}$ is an $q$-by- $q$ matrix. The transition matrix of the AMC of a supernode is

$$
\mathbf{P}=\left(\begin{array}{cccccccc}
1 & c_{0} & c_{1} & c_{2} & \ldots & \ldots & \ldots & c_{k_{\max }} \\
0 & 0 & d_{1} & 0 & \ldots & \ldots & \ldots & 0 \\
0 & b_{0} & 0 & d_{2} & 0 & \ldots & \ldots & 0 \\
0 & 0 & b_{1} & 0 & d_{3} & 0 & \ldots & 0 \\
\vdots & \vdots & \vdots & \vdots & \vdots & \vdots & \vdots & \vdots \\
0 & 0 & 0 & \ldots & \ldots & b_{k_{\max }-2} & 0 & d_{k_{\max }} \\
0 & 0 & 0 & \ldots & \ldots & 0 & b_{k_{\max }-1} & 0
\end{array}\right)
$$

where: $k_{\max }$ is the maximum node degree; $b_{k}$ is the probability of leaving state $k$ to go into state $k+1 ; d_{k}$ is the probability of leaving state $k$ to go into state $k-1$; and $c_{k}$ is the probability of leaving state $k$ to go into the absorbing state A.

The maximum node degree $k_{\max }$ is, in theory, $N_{s}-1$, where $N_{s}$ is the number of supernodes. In practice, a reasonable value $^{1}$ is $m+\mathbb{E}\left[L_{s}\right] \lambda_{s}$, where $\lambda_{s}$ (dimension: [nodes/min]) is the arrival rate of supernode's neighbors and can be expressed as

$$
\lambda_{s}=\lambda[1-F(d)] \frac{m}{\mathbb{E}\left[N_{s}\right]}
$$

where $\lambda$ is the average arrival rate of leaf nodes; $[1-F(d)]$ is the fraction of nodes which is promoted (i.e., with lifetime longer than $d$ ); the probability $m / \mathbb{E}\left[N_{s}\right]$ is due to the nonpreferential attachment strategy adopted by the supernodes. Since

$$
\mathbb{E}\left[N_{s}\right]=\lambda[1-F(d)] \mathbb{E}\left[L_{s}\right]
$$

from (5) and (6) if follows that $k_{\max } \simeq m+\mathbb{E}\left[L_{s}\right] \lambda_{s}=2 m$.

We recall that, for any AMC, being $V_{i}(n)$ the number of visits to the $i$-th transient state $(i \in\{1, \ldots, q\})$ in the discretetime interval $^{2}\{0, . ., n\}$, then

$$
\mathbb{E}[\mathbf{V}(n)]=\sum_{j=0}^{n} \mathbf{Q}^{j} \cdot \mathbf{p}(0)
$$

where, according to the proposed USN model,

$$
\mathbf{p}(0)=(0, \ldots, 0, \underbrace{1}_{m \text {-th position }}, 0, \ldots, 0) .
$$

Therefore, the expected number of visits of a supernode to state $k$ is

$$
\mathbb{E}\left[V_{k}(n)\right]=\sum_{j=0}^{n} q_{k m}^{(j)}
$$

where $q_{k m}^{(j)}$ is the $(k, m)$-th element of the matrix $\mathbf{Q}^{j}$.

The PMF of the node degree of a supernode at step $n$ can then be expressed as follows:

$$
P_{s}(k, n)=\frac{\mathbb{E}\left[V_{k}(n)\right]}{\sum_{l=0}^{k_{\max }} \mathbb{E}\left[V_{l}(n)\right]}=\frac{\sum_{j=0}^{n} q_{k m}^{(j)}}{\sum_{l=0}^{k_{\max }} \sum_{j=0}^{n} q_{l m}^{(j)}} .
$$

If there exists $m^{\prime}$ such that, $\forall m \geq m^{\prime}$, it holds that $P_{s}(0, n)=$ 0 (which is equivalent to $\mathbb{E}\left[V_{0}(n)\right]=0$ ), then $\mathbb{E}[T] \rightarrow \infty$, i.e. a supernode never becomes isolated. In Subsection III-A, the existence of such a value of $m$ will be shown.

\footnotetext{
${ }^{1}$ In fact, during the average lifetime $\mathbb{E}\left[L_{s}\right]$ as supernode, at most $\mathbb{E}\left[L_{s}\right] \lambda_{s}$ newly promoted supernodes can connect to the supernode as neighbors.

${ }^{2}$ Each discrete time instant corresponds to a state change in the AMC.
} 
At this point, we need to compute the transition probabilities of the AMC in order to evaluate the PMF of the node degree distribution. Denote as $R_{s}$ the residual lifetime of a supernode at its promotion. With reference to a supernode at a generic instant (after promotion), denote: its residual lifetime as $R_{s s}$; the residual lifetimes of its neighbors as $\left\{R_{n_{1}}, \ldots, R_{n_{k}}\right\}$; and as $A$ the time interval before the arrival of the next supernode neighbor. The one-step transition probabilities of the AMC in Fig. 1 can then be expressed as follows:

$$
\begin{aligned}
b_{k} & =P\left\{A<\min \left\{R_{s s}, R_{n_{1}}, . ., R_{n_{k}}\right\}\right\} \\
& =P\left\{A<R_{s s}\right\} P\left\{A<R_{n_{1}}\right\} . . P\left\{A<R_{n_{k}}\right\} \\
c_{k} & =P\left\{R_{s s}<\min \left\{A, R_{n_{1}}, . ., R_{n_{k}}\right\}\right\} \\
& =P\left\{R_{s s}<A\right\} P\left\{R_{s s}<R_{n_{1}}\right\} . . P\left\{R_{s s}<R_{n_{k}}\right\} \\
d_{k} & =1-b_{k}-c_{k} .
\end{aligned}
$$

Since the residual lifetimes of different supernodes are independent and have the same distribution, by defining $p$ as $P\left\{R_{x}>A\right\} \quad\left(x \in\left\{s s, n_{1}, \ldots, n_{k}\right\}\right)$ and observing that $P\left\{R_{x}<R_{y}\right\}=1 / 2\left(x, y \in\left\{n_{1}, \ldots, n_{k}\right\}, x \neq y\right)$, it follows that

$$
\begin{aligned}
b_{k} & =p^{k+1} \\
c_{k} & =(1 / 2)^{k}(1-p) \\
d_{k} & =1-p^{k+1}-(1 / 2)^{k}(1-p) .
\end{aligned}
$$

Therefore, it is sufficient to evaluate $p$ to fully characterize the AMC.

The following theorem shows that, in the scenario of interest (leaf nodes have Poisson arrivals and shifted Pareto lifetimes), $p$ can be approximated with an expression which does not depend on $d$ and $\lambda$.

Theorem 2. Suppose that (i) the inter-arrival time (into the network) of leaf nodes has an exponential distribution with parameter $\lambda$ (Poisson arrivals) and (ii) a leaf's node lifetime $L$ has a shifted Pareto CDF equal to $F(x)=1-(1+x / \beta)^{-\alpha}$, $x>0, \alpha>1, \beta>0$. Assuming that a leaf node with $L>d$ is promoted to a supernode after $d$ time units, attaching non-preferentially to $m$ already present supernodes, the characteristic parameter $p$ of the supernode's AMC model can be approximated as follows:

$p \simeq 1-(\alpha-1)(\beta m / \mathbb{E}[L])^{\alpha-1} e^{\beta m / \mathbb{E}[L]} \Gamma(-\alpha+1, \beta m / \mathbb{E}[L])$

being $\Gamma(a, b) \triangleq \int_{b}^{+\infty} t^{a-1} e^{-t} \mathrm{~d} t$ the incomplete gamma function.

Proof: As shown above, the average arrival rate $\lambda_{s}$ of a supernode's neighbors is given by (5). By approximating the distribution of the arrival process of a supernode's neighbors as Poisson $\left(\lambda_{s}\right)$, the distribution of the residual time $A$ before the arrival of the next supernode neighbor can be approximated as exponential with parameter $\lambda_{s}$, i.e.,

$$
F_{A}(x) \simeq 1-e^{-\lambda_{s} x} \quad x \geq 0 .
$$

Similarly to (1), the distribution of the residual lifetime of a supernode (after promotion) can be written as

$$
F_{R_{s}}(x)=\frac{1}{\mathbb{E}\left[L_{s}\right]} \int_{0}^{x}\left[1-F_{s}(y)\right] \mathrm{d} y \quad x \geq 0
$$

By using Theorem 1 and using the same substitution strategy used in its proof to solve the integral above, one obtains

$$
F_{R_{s}}(x)=1-\left(\frac{\beta+d}{\beta+x+d}\right)^{\alpha-1} \quad x \geq 0 .
$$

Therefore, taking into account that $A$ and $R_{s}$ are independent and using (5) and (6), it follows:

$$
\begin{aligned}
p & =P\left\{R_{s}-A<0\right\}=\int_{-\infty}^{+\infty} \int_{-\infty}^{y} f_{A R_{s}}(x, y) \mathrm{d} x \mathrm{~d} y \\
& =\int_{-\infty}^{+\infty} f_{R_{s}}(y) \int_{-\infty}^{y} f_{A}(x) \mathrm{d} x \mathrm{~d} y=\int_{-\infty}^{+\infty} f_{R_{s}}(y) F_{A}(y) \mathrm{d} y \\
& \simeq C \int_{0}^{+\infty}(1+(y+d) / \beta)^{-\alpha}\left(1-e^{-\lambda_{s} y}\right) \mathrm{d} y
\end{aligned}
$$

where $C \triangleq\left[(\alpha-1)(1+d / \beta)^{\alpha-1}\right] / \beta$. With some simple substitutions and taking into account the expression of the incomplete gamma function, one can show that

$p \simeq 1-(\alpha-1)\left((\beta+d) \lambda_{s}\right)^{\alpha-1} e^{(\beta+d) \lambda_{s}} \Gamma\left(-\alpha+1,(\beta+d) \lambda_{s}\right)$.

The proof is completed by making the following substitution in the previous expression of $p:(\beta+d) \lambda_{s}=(\beta+d) m /(1+$ $d / \beta) \mathbb{E}[L])=\beta m / \mathbb{E}[L]$.

\section{Generalizations}

The arrival process of a supernode's neighbors is not Poisson, as assumed in the proof of Theorem 2: in fact, some of the leaf nodes, which arrive with Poisson distribution with parameter $\lambda$, die before being promoted to supernodes. The derivation of the exact distribution of the arrival process of a supernode's neighbors and, therefore, of the distribution of $A$ and of an exact expression for $p$, is an open problem.

Moreover, other attachment strategies, besides nonpreferential, may be adopted. For example, the attachment strategy could privilege supernodes which are more powerful (in terms of resources) or have longer lifetimes. In this case, the resulting node degree distribution is likely to be characterized by a power law [9] and the extension of our framework in this direction is interesting. For instance, with a preferential attachment strategy the average arrival rate of a supernode's neighbors, now completely characterized by $\lambda_{s}$ in (5), would require to take into account the node degree of the specific supernode [10]. Obviously, this complicates the framework.

Another interesting generalization of our framework is related to a scenario where a peer can indefinitely change its status from supernode to leaf, and vice versa. In this case, new expressions for $F_{s}(x)$ and $E\left[L_{s}\right]$ would be derived.

\section{Performance Analysis}

\section{A. Analytical vs Simulation Results}

We have simulated 7 days of the life of an USN, where leaf nodes are have inter-arrival time modeled by an exponential distribution with parameter $\lambda=34.8$ nodes/min - this value corresponds to the value of 0.58 nodes/s obtained in [5]. Leaf nodes are promoted to the supernode state if their session length $L$ exceeds $d=3$ hours. $L$ has a shifted Pareto 


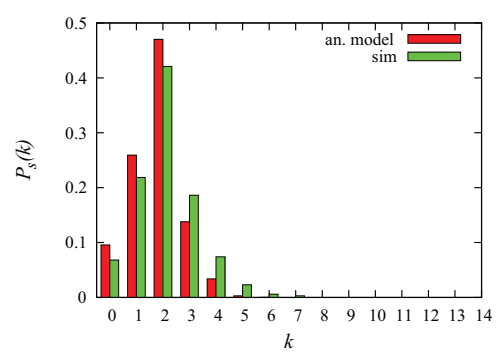

(a)

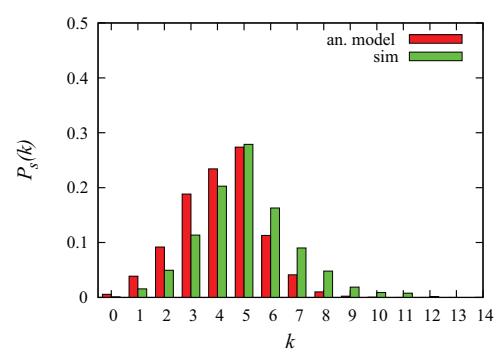

(b)

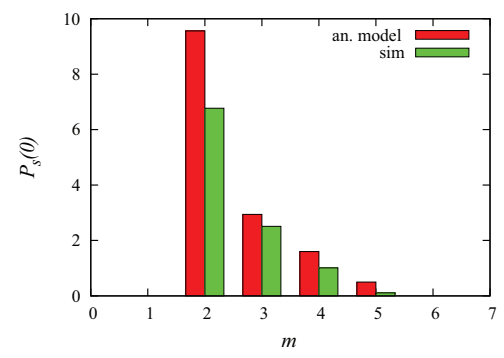

(c)

Fig. 2. Steady-state USN scenario with $\alpha=3, \beta=60, \mathbb{E}[L]=30$ : in (a)-(b), node degree distributions of supernodes with $m=2$ and $m=5$, respectively; in (c), fraction of isolated supernodes as a function of $m$.

distribution with $\alpha=3$ and $\beta=60$, so that $\mathbb{E}[L]=30$ minsthis corresponds to a realistic value according to [2]. After a transient period of about 14 hours, the network reaches a "stable" size, with on average $\lambda \mathbb{E}[N]=\lambda \mathbb{E}[L]=1044$ nodes, of which $6 \%$ are supernodes. The average lifetime of supernodes is 120 minutes, as predicted by equation (3).

In Figs. 2 (a) and (b), the node degree PMFs of the supernodes are shown for $m=2$ and $m=5$, respectively. For each value of $m$, the analytical results predicted by the proposed framework (with $k_{\max }=15$ and $n=2 \mathrm{~m}$ ) are directly compared with simulation results. It can be observed that the agreement is good. The numerical solutions of the analytical model have been obtained with $n=2 m$ instead of $n \rightarrow \infty$ because the former is the realistic number of state changes over the AMC. ${ }^{3}$ We discuss this aspect further in the following Subsection III-B.

In Fig. 2 (c), the probability of supernode isolation (i.e., $P_{s}(0)$ ) is shown as a function of $m$. In the simulation case, this probability is evaluated as the percentage of isolated supernodes, with respect to the total number of promoted supernodes, after the entire simulation duration (7 days). As for the evaluation of the PMF, in this case as well a good agreement between simulation and analytical results can be observed. In particular, the proposed framework allows to evaluate very effectively the resilience of an USN by simply evaluating $P_{s}(0)$.

\section{B. Discussion}

As we observed in Subsection II-C, the sojourn time in a given state of the AMC, i.e., the time interval during which a supernode has a given number of neighbors, is not exponentially distributed. Nevertheless, the AMC-based approach allows to derive a PMF of the node degree which approximates well the simulation-based (realistic) PMF, provided that a proper value is assigned to the number $n$ of state changes before absorption. Our analysis indicates that $n=2 m$ is the optimized value- - this is expected, as the average numbers of supernodes' arrivals and departures, during $\mathbb{E}\left[L_{s}\right]$, are both equal to $\mathbb{E}\left[L_{s}\right] / \lambda_{s}=m$.

From the results in Fig. 2 (c), we see that the analytical approach predicts an isolation probability $P_{s}(0)$ slightly higher than the value given by the simulation. However, both analysis and simulations lead to the conclusion that selecting a value $m \geq 6$ guarantees, in the considered scenario, that $P_{s}(0)=0$,

\footnotetext{
${ }^{3}$ Other simulation results, not shown here for lack of space, indicate that the real number of state changes, evaluated with simulations, is approximately $2 m-0.3$.
}

i.e., there is no supernode isolation-for other scenarios, the minimum value of $m$ for no supernode isolation might be different.

\section{CONCLUSION}

In this letter, we have illustrated a novel analytical framework for the evaluation the resilience of USNs. The supernode behavior, in terms of connections with other supernodes, has been described by a proper AMC, fully characterized by a single parameter $p$, for which a simple approximate expression has been derived. The proposed framework leads to the derivation of the minimum number of connections $m$ that a supernode must activate, at its promotion, towards other supernodes in order to avoid isolations. Simulation results, related to a realistic scenario, confirm the analytical ones. In general, depending on the application(s) running over a P2P network, the parameters which allow to compute the minimum value of $m$ may change over time. Therefore, the minimum value of $m$ required for no node isolation should be dynamically adjusted, e.g., using gossiping strategies to propagate value updates. It is important to underline that nothing prevents supernodes from establishing a number of connections $m$ much larger than the minimum one-for instance, to be robust against sudden churn rate variations and network performance issues. A practical implementation of the proposed system is currently under investigation.

\section{REFERENCES}

[1] E. K. Lua, J. Crowcroft, M. Pias, R. Sharma, and S. Lim, "A survey and comparison of peer-to-peer overlay network schemes," IEEE Commun. Surveys \& Tutorials, vol. 7, no. 2, pp. 72-93, 2005.

[2] D. Leonard, Z. Yao, V. Rai, and D. Loguinov, "On lifetime-based node failure and stochastic resilience of decentralized peer-to-peer networks," IEEE/ACM Trans. Netw., vol. 15, no. 3, pp. 644-656, June 2007.

[3] M. Amoretti, "A modeling framework for unstructured supernode networks," IEEE Commun. Lett., vol. 16, no. 10, pp. 1707-1710, Oct. 2012.

[4] D. Stutzbach and R. Rejaie, "Understanding churn in peer-to-peer networks," in Proc. 2006 ACM SIGCOMM Conference on Internet Measurement, pp. 189-202.

[5] D. Ilie and A. Popescu, "Statistical models for gnutella signaling traffic," Computer Networks, vol. 51, no. 17, pp. 4816-4835, Dec. 2007.

[6] F. E. Bustamante and Y. Qiao, "Friendships that last: peer lifespan and its role in $\mathrm{p} 2 \mathrm{p}$ protocols," in Web Content Caching and Distribution, F. Douglis and B. D. Davison, editors. Kluwer Academic Publishers, 2004, pp. 233-246.

[7] S. I. Resnick, Adventures in Stochastic Processes. Birkhauser, 2002.

[8] G. Bolch, S. Greiner, H. D. Meer, and K. S. Trivedi, Queueing Networks and Markov Chains. Wiley-Interscience, 2005.

[9] B. Yang and H. Garcia-Molina, "Designing a super-peer network," in Proc. 2003 Int.'l Conference on Data Engineering, pp. 49-60.

[10] R. Albert and A.-L. Barabasi, "Statistical mechanics of complex networks," Rev. Mod. Physics, vol. 74, no. 1, pp. 47-92, Jan. 2002. 